\title{
The spectrum dip of deck mounted systems
}

\author{
R.J. Scavuzzo ${ }^{\mathrm{a}, *}$, G.D. Hill ${ }^{\mathrm{b}}$ and P.W. Saxe ${ }^{\mathrm{b}}$

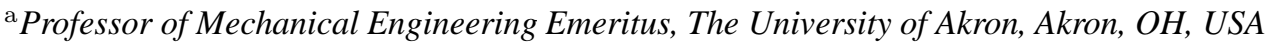 \\ ${ }^{\mathrm{b}}$ Alion Science and Technology, 4300 King St., Suite 101, Alexandria, VA, USA
}

Received 19 March 2008

\begin{abstract}
In this paper, a detailed model of a ship deck and attached dynamic systems was developed and subjected to dynamic studies using two different shock inputs: a triangular shaped velocity pulse and the vertical motion of the innerbottom of the standard Floating Shock Platform (FSP). Two studies were conducted, one considering four single degree-of-freedom systems attached at various deck locations and another considering a three-mass system attached at one location. The two shock inputs were used only for the multi-mass system study. The triangular pulse was used for the four single degree-of-freedom systems study. For the single degree-of-freedom systems study, shock spectra were first calculated at the four mounting locations assuming the oscillators were not present. Then the oscillator systems were added to these grid points to determine the change in the shock spectra. First, the oscillators were added one at a time, and then all the oscillators were added to the deck. The multi-mass system was analyzed using both shock inputs. First, the fixed-base modal masses and frequencies were determined. Then, the system as a whole was attached to the deck and the spectrum values at the base point were determined and compared to those for the free deck case. In the last step each mode of the multi-mass system, represented by a single degree-of-freedom system with the modal mass and appropriate spring stiffness, was considered individually to determine the spectrum responses. Results of the free deck, the entire system and individual modal responses are compared.
\end{abstract}

Keywords: Spectrum dip, DDAM, ship shock

\section{Introduction}

In order to determine the dynamic response motion of a structure supported at its base, input motion in the form of acceleration, velocity or displacement at the base of the system must be known. The spectral response of the structure is the maximum relative displacement between a single degree-of-freedom system at a particular frequency subjected to this motion and the base. If the base structure is flexible as in the deck or hull of a ship, or even the earth, dynamic interaction will alter the base input motion from its free, or unloaded, motion. The shock spectrum of this altered motion will be reduced, sometimes very significantly. Often the resulting shock or response spectrum of the base motion is used as the dynamic input to the equipment structure rather than the measured time-history motion. In these analyses, the normal modes of the equipment structure are found at the base of that structure where it is fixed at the deck of a ship or foundation. The shock spectrum inputs are specified for each of these fixed-base normal modes.

Since the structure is mounted on a flexible base, the frequencies calculated assuming a fixed-base will not be the actual vibration modes of the entire structure. However, these spectrum inputs are specified at the frequencies of the vibration modes determined at the fixed-base of the system, which is the connection or connections of the equipment structure to the deck or earth. It is spectrum input at the fixed-base frequencies only that specify dynamic loads to the structure. Thus, the shock spectrum has input only at the fixed-base modes of vibration and not the actual modes of vibration of the entire deck/structure system. Conversely, the dynamic forces from the equipment back to the

\footnotetext{
*Corresponding author. E-mail: RSCRUD@aol.com.
} 
Node 700 (Seismic Mass)

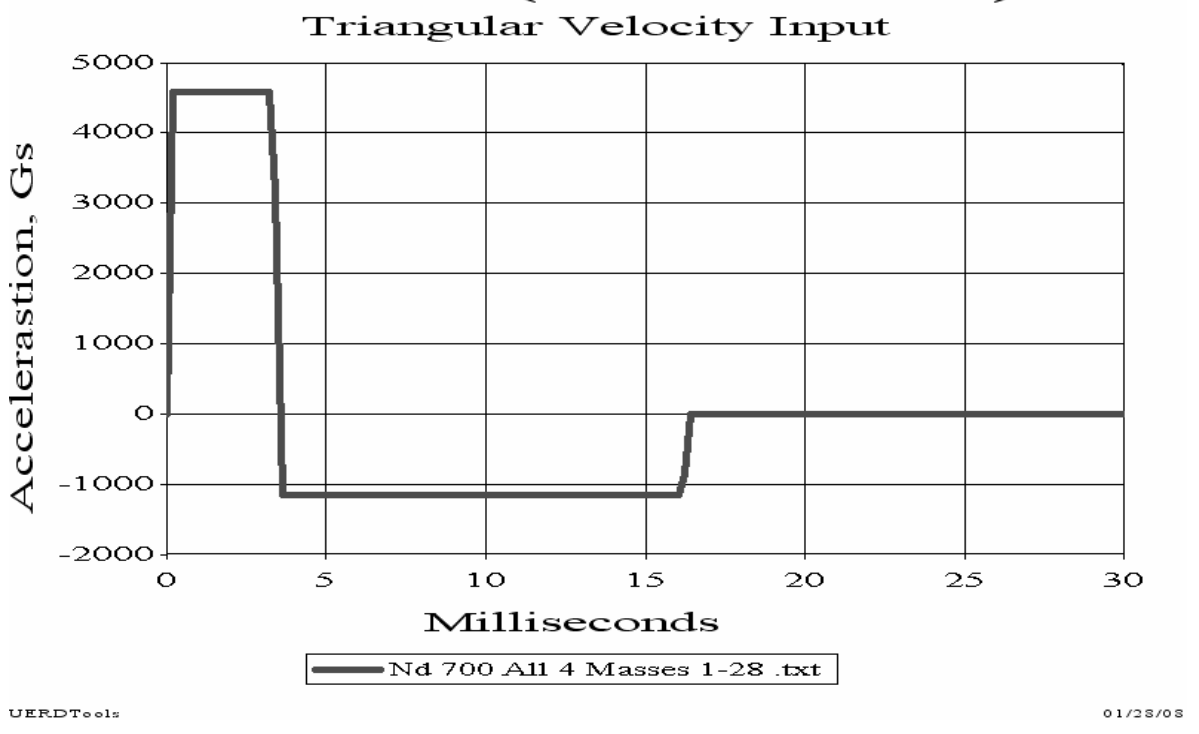

Fig. 1. Acceleration motion (Gs) of the triangular velocity shock at Node 700.

deck occur only at the fixed-base vibration frequencies [4-6]. Thus, the input dynamic motion measured at the deck is suppressed the most at the fixed-base frequencies of the structure.

Prior to the understanding of the input motion suppression, or spectrum dip effect, an envelope of shock or response spectrum was considered for design, especially in earthquake engineering [3,7]. In ship shock, the resulting shock spectrum inputs are up to an order of magnitude too high if interaction affects are not considered $[1,4,5,8,9]$. In earthquake engineering the change is much smaller [7].

It is the purpose of this paper to investigate two types of dynamic systems mounted on a flexible base structure. First, four single degree-of-freedom systems mounted to a typical ship deck are considered. The objective of examining these single-mass systems is to evaluate the reduction in the deck shock spectrum with oscillator weight and also with proximity of the oscillators to the measurement point and to each other. Second, a three-mass system is considered in which the influence of the entire dynamic system on the higher modes, with lower modal masses, is studied. Results of this analysis have implications for the US Navy Dynamic Design Analysis Method (DDAM) of properly accounting for the effect of interaction of modal masses in a system on each other. Inputs to the higher modes, usually with lower modal masses, are based on the modal weight of that higher mode only. Using the three-mass model, the effect of the total system on the shock input to the higher modes can be evaluated. Results indicate that DDAM inputs may be too high.

\section{Input motions}

Two input motions were used in this study of interaction of dynamic systems with a flexible deck: a triangular velocity pulse and the inner-bottom motion of the US Navy standard Floating Shock Platform (FSP). Both input motions were imposed on a seismic mass, Node 700, of the mathematical model. A triangular velocity shaped shock is often observed at the keel of surface combatants when subjected to underwater shock. However, the pulse in this study is much different in magnitude, acceleration and deceleration than typical measured values. Since the objective of the study is to evaluate the differences in the response of the deck with and without dynamic systems, the magnitude and exact shape do not have to be accurate. The FSP measured motion is available in unclassified published literature. So the motion in velocity and acceleration is specified.

The prescribed acceleration for the triangular velocity input is presented in Gs, and the acceleration of the FSP deck is in in $/ \mathrm{sec}^{2}$. Graphs of the two motions in acceleration and velocity are shown in Figures 1-4. The shape of 
Node 700 (Seismic Mass)

Triangular Velocity Input

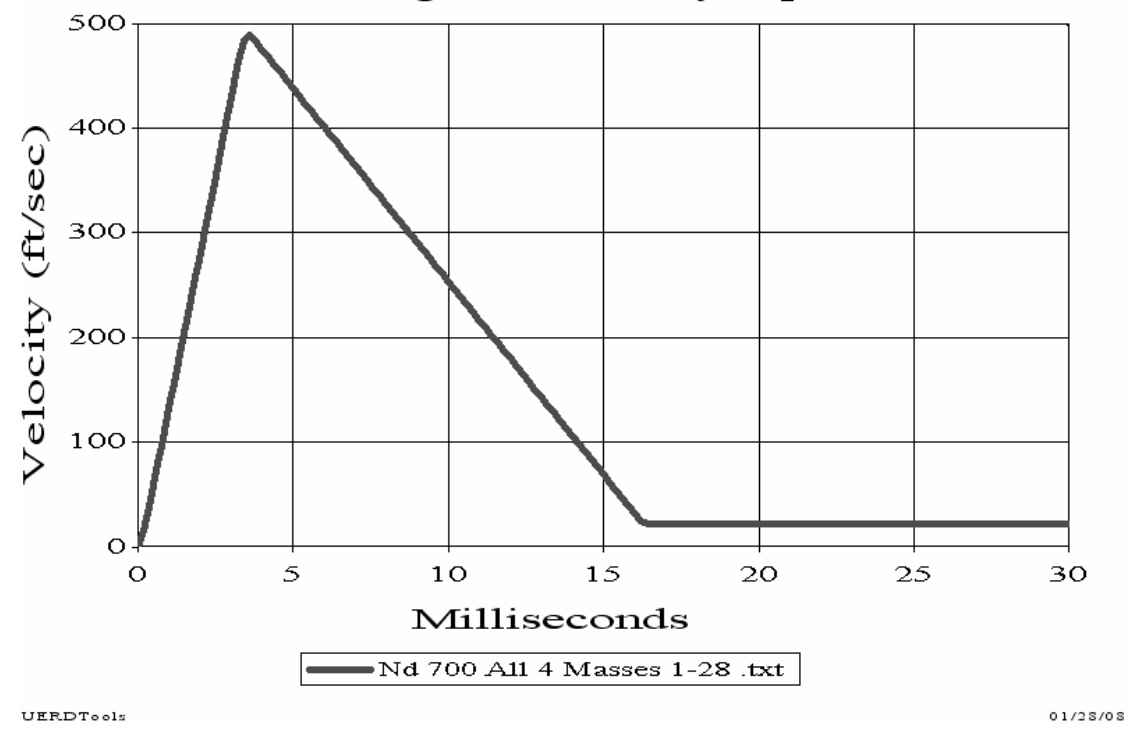

Fig. 2. Triangular velocity shock (ft/sec) at Node 700 .

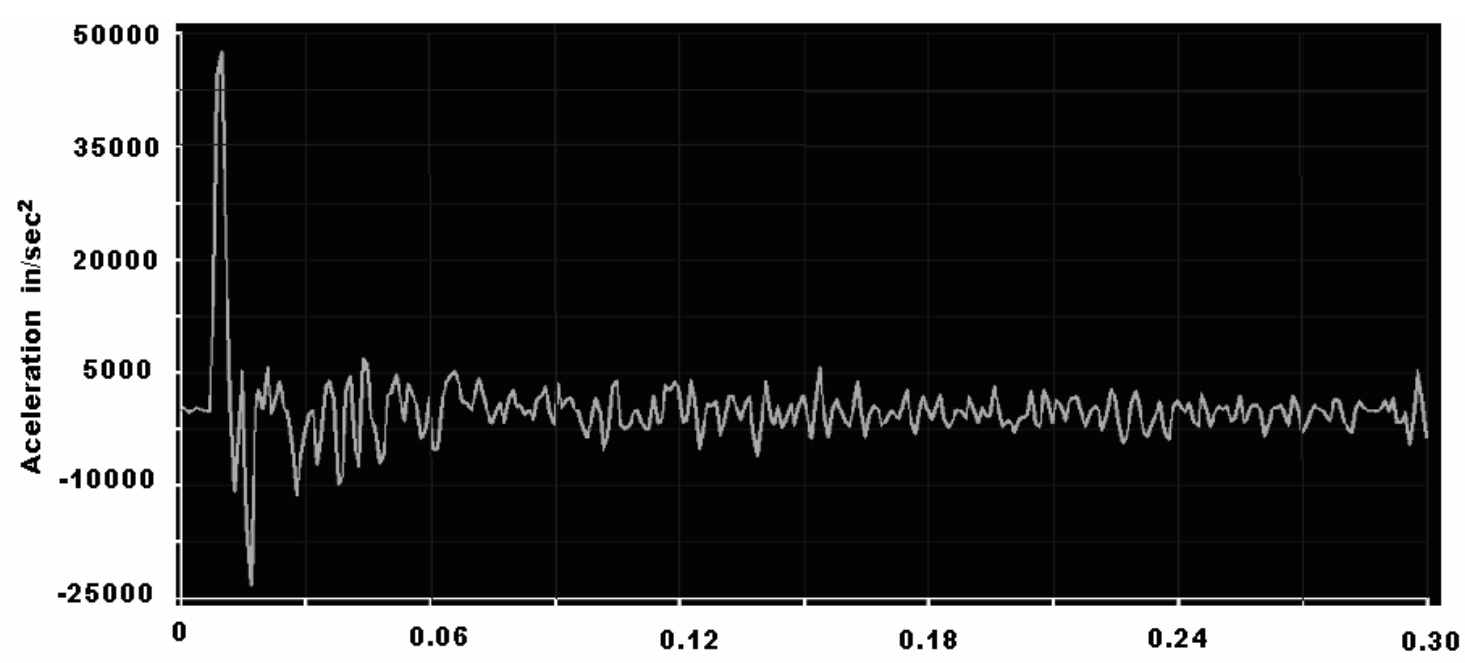

Fig. 3. Acceleration motion $\left(\mathrm{in} / \mathrm{sec}^{2}\right)$ of the FSP shock.

the velocity pulse is similar to measured motions at the ship keel and was therefore used to evaluate the inputs to both types of dynamic systems. The FSP motion was applied to evaluate if there were any basic differences using a more complex acceleration motion.

\section{Ship deck characteristics}

The authors modeled a section of a typical navy ship deck, using NEiNastran Modeler which is a licensed version of Femap, to evaluate the effects of dynamic interaction. The actual calculations were made using NEiNastran Editor using the model developed in the Modeler. Top and bottom views of the deck model are shown in Figs 5 and 6 , 


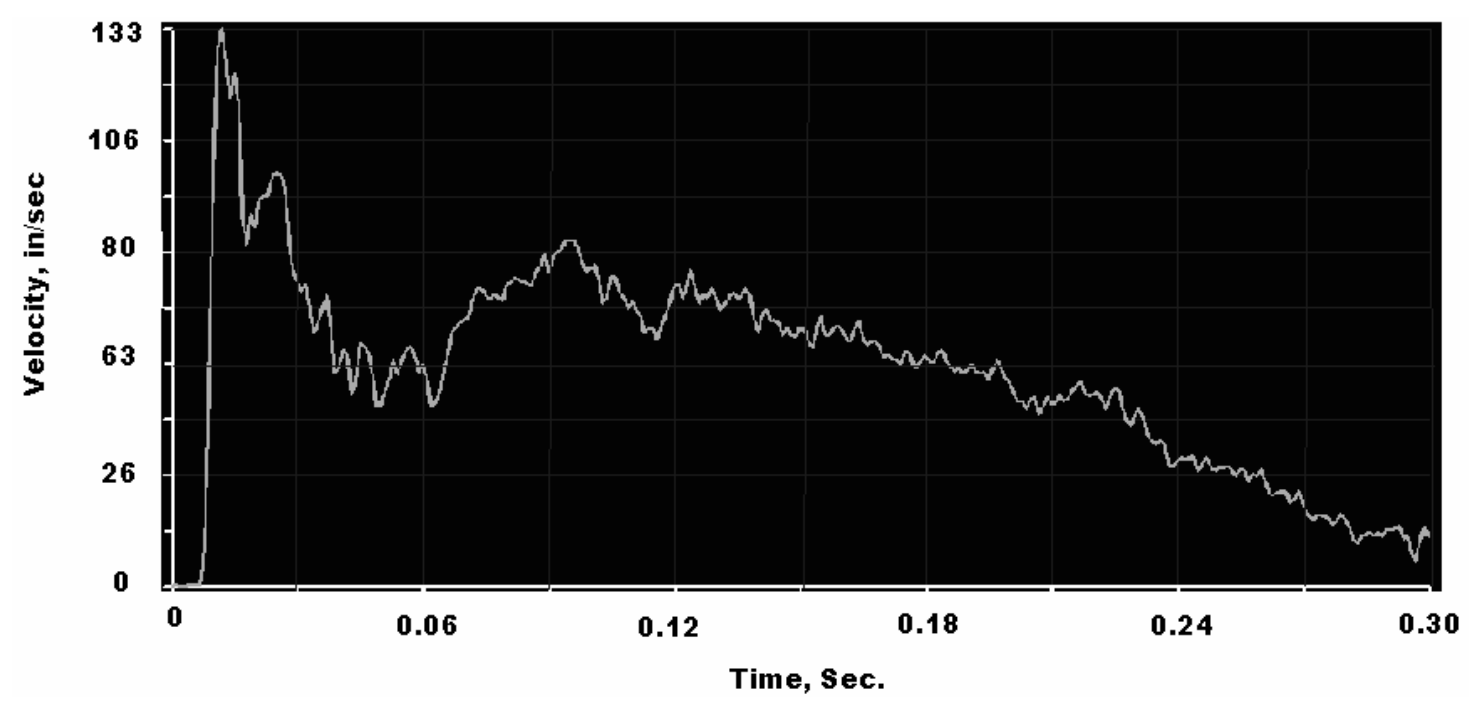

Fig. 4. Velocity motion (in/sec) of the FSP shock.

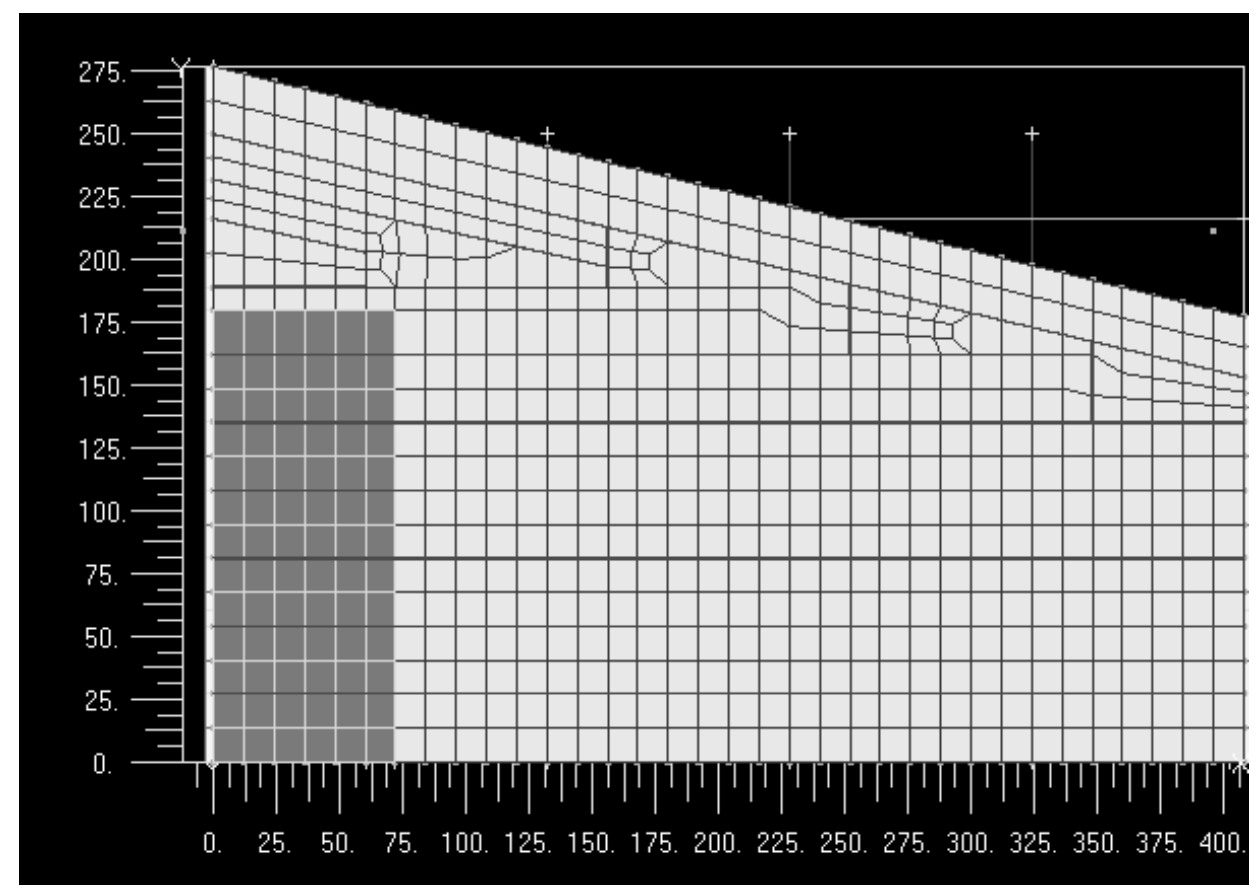

Fig. 5. Top view of the deck model used in the study.

respectively. In typical decks, there are deep transverse tee-beams supporting less stiff longitudinal beams as shown in Fig. 6. The longitudinal edges of the deck model were reinforced with rigid weightless beam elements to prevent low frequency edge vibration modes (Fig. 7). The shapes of the first 6 modes of vibration of the free deck with edge reinforcements are shown in Fig. 8. The frequencies of modes 1-10 are listed in Table 1.

\section{Dynamic systems}

Two different groups of dynamic systems attached to the deck were considered in the investigation: 


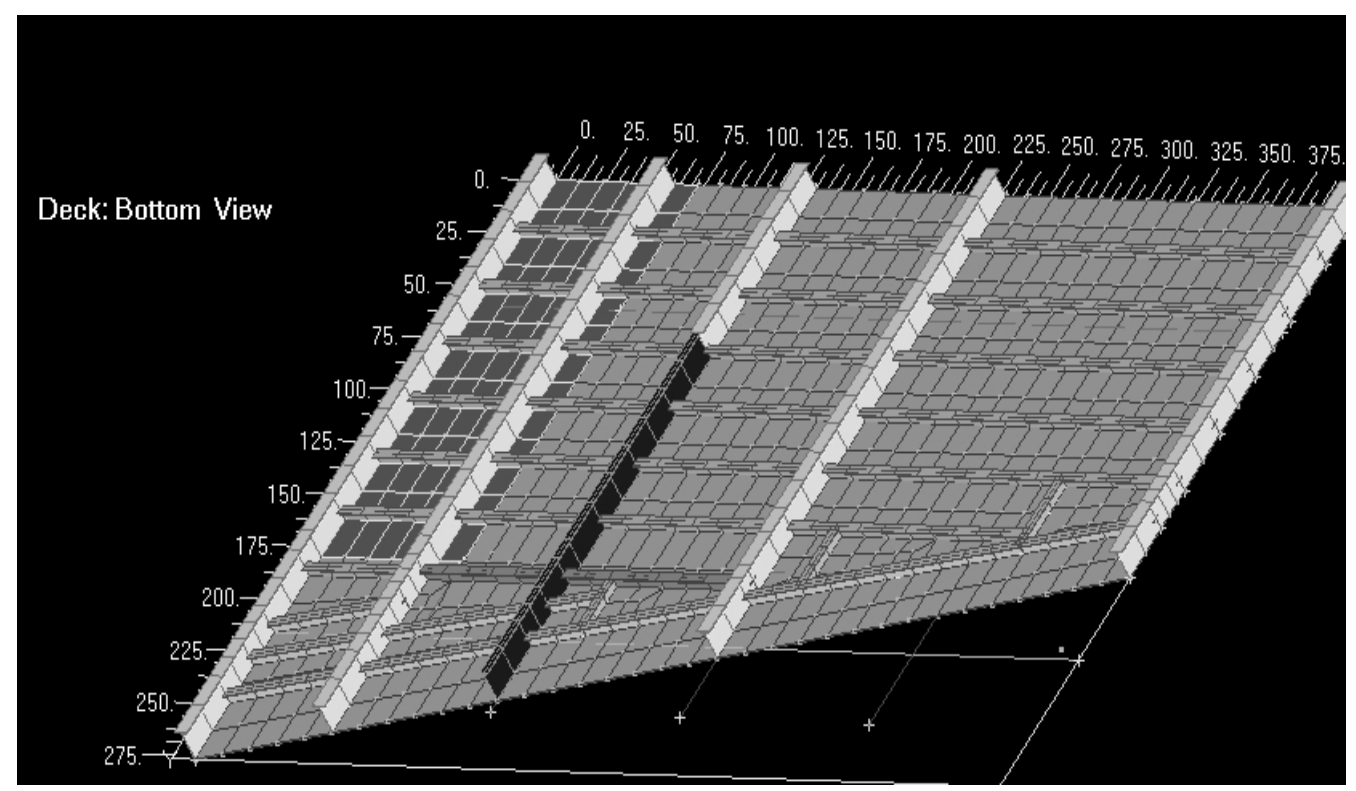

Fig. 6. Bottom view of the deck model used in the study showing main transverse beams, and longitudinal reinforcement.

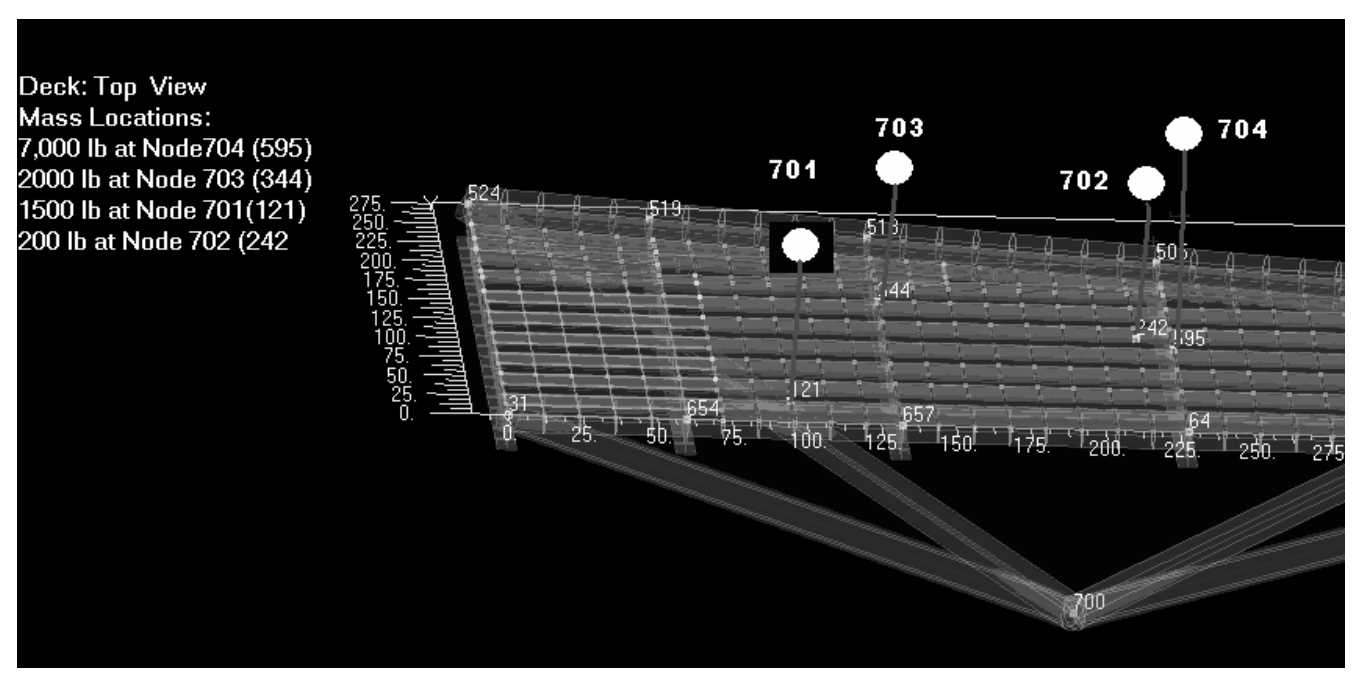

Fig. 7. Weightless reinforcements were added to the deck to represent longitudinal bulkheads, shell, and connections to the driving seismic mass at Node 700. Nodes 701 thru 704 are locations of concentrated masses connected to the deck by vertical springs. Motions of these masses are constrained except in the vertical direction.

1. Four single degree-of-freedom systems at four locations as indicated in Figs 7 and 9 and in Table 2. Masses ranged from $200 \mathrm{lbm}$ to $7,000 \mathrm{lbm}$ with fixed-base frequencies from $22 \mathrm{~Hz}$ to $30 \mathrm{~Hz}$. This system was subjected to the triangular velocity shock only.

2. A three-mass system with a total mass of 7,000 lbm fixed to Node 595 with modal fixed-base frequencies of $31 \mathrm{~Hz}, 60 \mathrm{~Hz}$ and $83 \mathrm{~Hz}$ (Tables 3 and 4 and Fig. 10). The three-mass system was subjected to both the triangular velocity shock and to the FSP shock.

Since lumped masses are used to evaluate dynamic interaction with the modeled ship deck, masses were constrained to have motion in the vertical direction only with respect to the deck. The deck is flexible in the vertical direction and therefore is expected to have significant dynamic interaction and is the most meaningful configuration to evaluate 


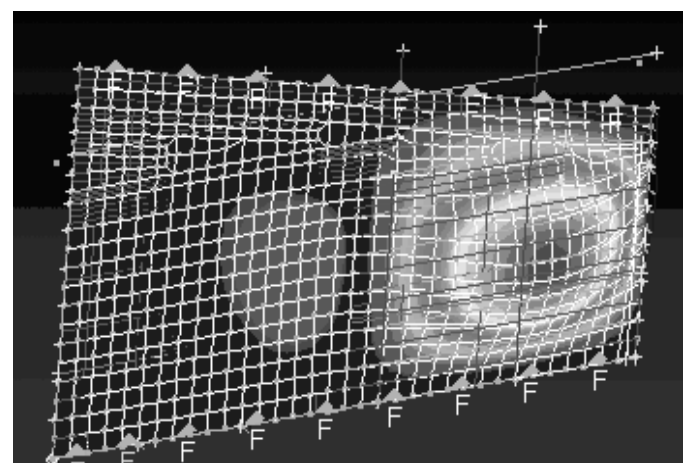

Mode $116.2 \mathrm{~Hz}$

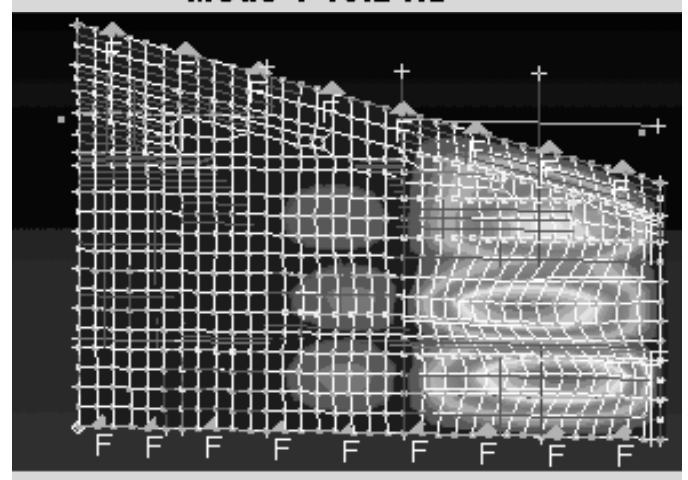

Mode $320.5 \mathrm{~Hz}$

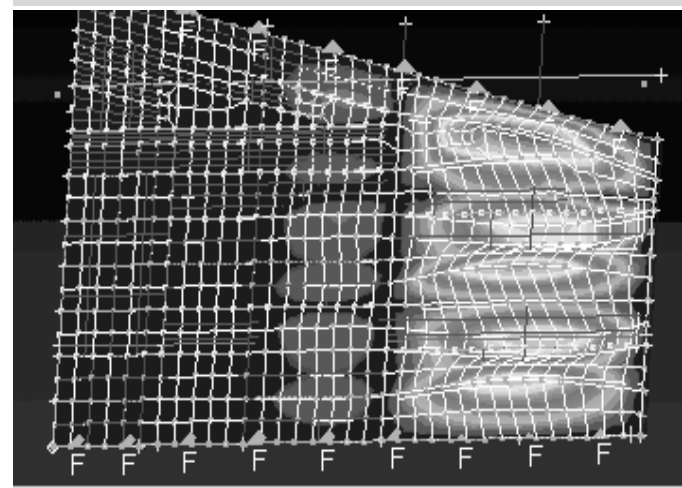

Mode $526.9 \mathrm{~Hz}$

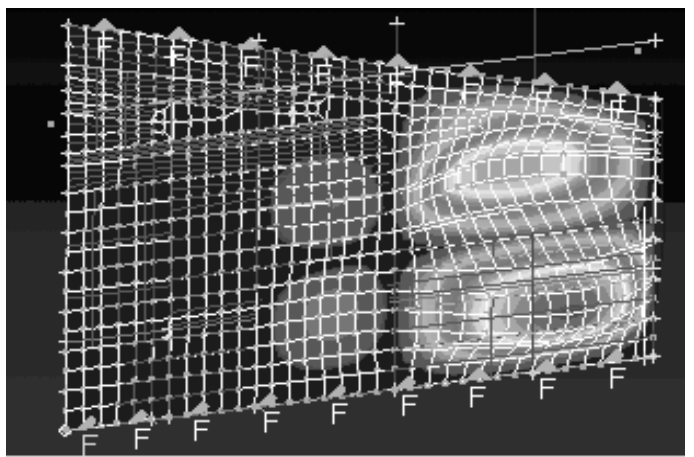

Mode $218.3 \mathrm{~Hz}$

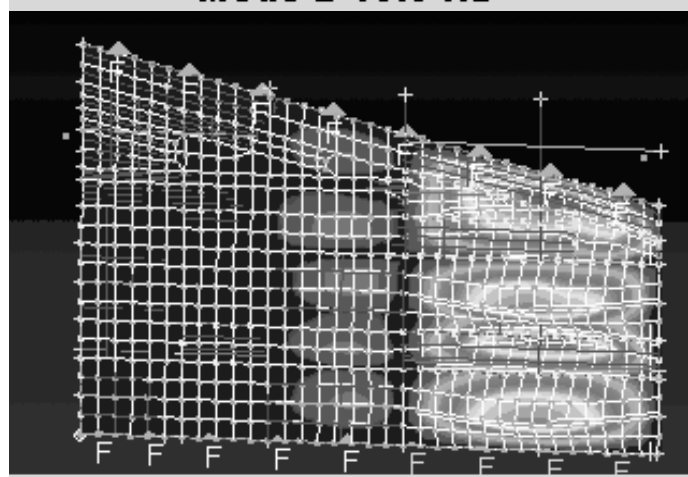

Mode $423.7 \mathrm{~Hz}$

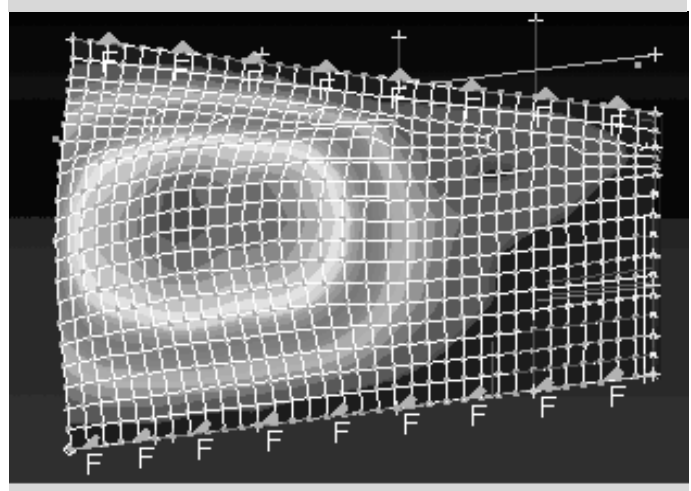

Mode $630.3 \mathrm{~Hz}$

Fig. 8. Deck Modes 1-6 with frequencies $16.2 \mathrm{~Hz}$ to $30.3 \mathrm{~Hz}$.

the spectrum dip phenomenon. Lateral interaction will be significant in other locations of the ship not examined in this paper.

\section{Dynamic calculations - four single degree-of-freedom systems}

The investigation was conducted by first determining the shock spectrum curves at the four node points to be used for spring attachment points when the deck was subjected to the triangular shock pulse. First, the deck only (free deck) model was considered. The time-history motion of these nodes is plotted in Fig. 11. The effect on the time-history motion with a $1,500 \mathrm{lb}$ weight supported at Node 121 with a $22 \mathrm{~Hz}$ fixed-base frequency is plotted in Fig. 12. Note the reduction in the peak acceleration compared to the free deck case near 0.0025 seconds at grid point 
Table 1

Frequencies of the deck model without added spring/mass systems

\begin{tabular}{cccc}
\hline Mode & Eigenvalue & Frequeny-Radians $(1 / \mathrm{sec})$ & Frequency-Cycles $(\mathrm{Hz})$ \\
\hline 1 & $1.020 \mathrm{E}+04$ & $1.017 \mathrm{E}+02$ & $1.619 \mathrm{E}+01$ \\
2 & $1.319 \mathrm{E}+04$ & $1.151 \mathrm{E}+02$ & $1.831 \mathrm{E}+01$ \\
3 & $1.653 \mathrm{E}+04$ & $1.287 \mathrm{E}+02$ & $2.049 \mathrm{E}+01$ \\
4 & $2.216 \mathrm{E}+04$ & $1.491 \mathrm{E}+02$ & $2.372 \mathrm{E}+01$ \\
5 & $2.848 \mathrm{E}+04$ & $1.690 \mathrm{E}+02$ & $2.689 \mathrm{E}+01$ \\
6 & $3.331 \mathrm{E}+04$ & $1.905 \mathrm{E}+02$ & $3.031 \mathrm{E}+01$ \\
7 & $4.312 \mathrm{E}+04$ & $2.114 \mathrm{E}+02$ & $3.364 \mathrm{E}+01$ \\
8 & $4.461 \mathrm{E}+04$ & $2.118 \mathrm{E}+02$ & $3.371 \mathrm{E}+01$ \\
9 & $6.141 \mathrm{E}+04$ & $2.481 \mathrm{E}+02$ & $3.948 \mathrm{E}+01$ \\
10 & $6.358 \mathrm{E}+04$ & $2.522 \mathrm{E}+02$ & $4.014 \mathrm{E}+01$ \\
\hline
\end{tabular}

Table 2

Single DOF dynamic masses on the deck model

\begin{tabular}{ccccc}
\hline Weight $(\mathrm{lbs})$ & Mass Node $^{*}$ & Deck Node & Freq. (Hz) & K (lb/in) \\
\hline 7000 & 704 & 595 & 30 & 644337 \\
2000 & 703 & 344 & 25 & 127845 \\
1500 & 701 & 121 & 22 & 74252 \\
200 & 702 & 242 & 23 & 10821 \\
\hline
\end{tabular}

*All degrees of freedom except vertical translation are constrained for all masses.

${ }^{* *}$ The listed frequency is the fixed-base frequency.

Table 3

Multimass system model characteristics

\begin{tabular}{lcccc}
\hline Position & Mass Node & Weight $(\mathrm{lb})$ & Mass $\left(\mathrm{lb}-\mathrm{s}^{2} /\right.$ in $)$ & Stiffness $(\mathrm{lb} / \mathrm{in})$ \\
\hline Lower & 801 & 4000 & 10.35 & 1178640 \\
Middle & 802 & 2000 & 5.18 & 589320 \\
Upper & 803 & 1000 & 2.59 & 294660 \\
\hline
\end{tabular}

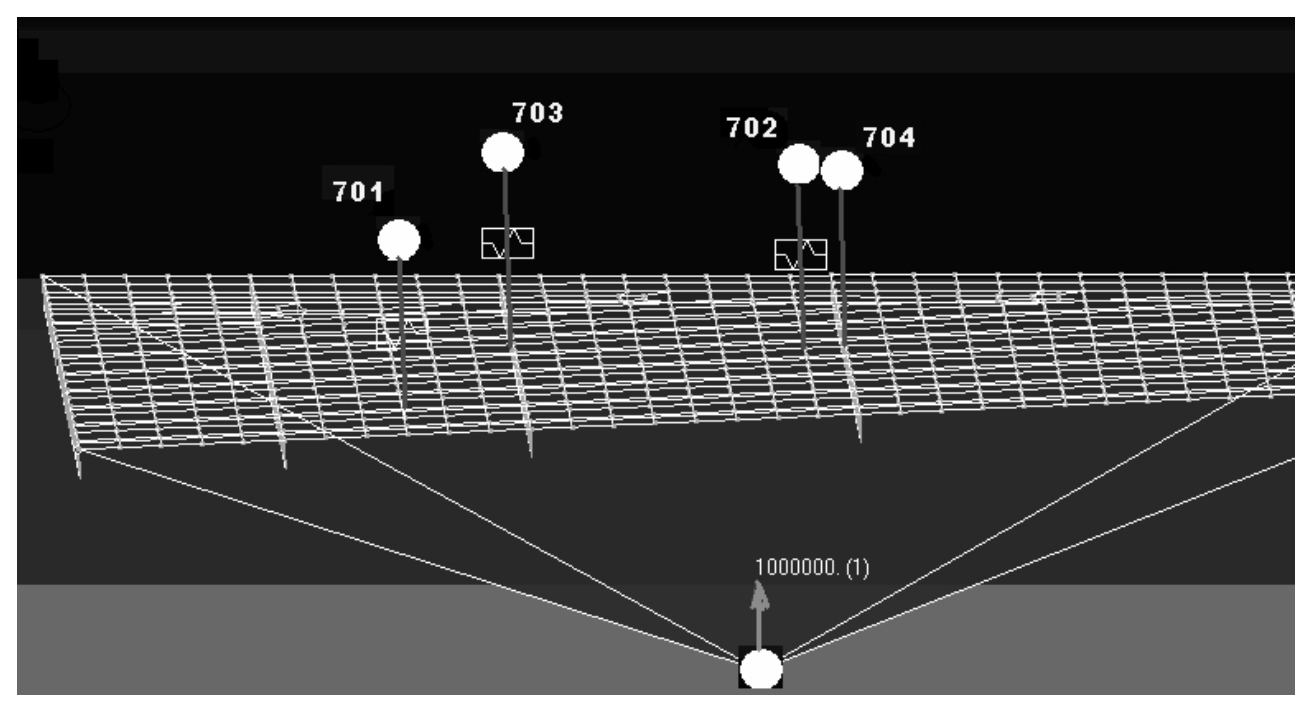

Fig. 9. Model showing the vertical springs and masses. Frequencies, weights, and grid points are listed in Table 2.

121 in Figs 11 and 12. The reduction in the time-history acceleration is caused by the dynamic interaction between the oscillator and the deck structure. The change in the time-history motion reduces the shock spectrum, with the largest change occurring near the fixed-base frequency. 
Table 4

Multi-mass system dynamic characteristics

\begin{tabular}{ccccc}
\hline Mode & Freq. $(\mathrm{Hz})$ & Modal Weight (lb) & Modal Mass (lb-s $\left.{ }^{2} / \mathrm{in}\right)$ & Modal Stiffness (lb/in) \\
\hline 1 & 30.8 & 5456 & 14.12 & 528805 \\
2 & 60.3 & 1315.3 & 3.404 & 488634 \\
3 & 83.3 & 225.73 & 0.5842 & 160034 \\
& Check & 6997 & & \\
\hline
\end{tabular}

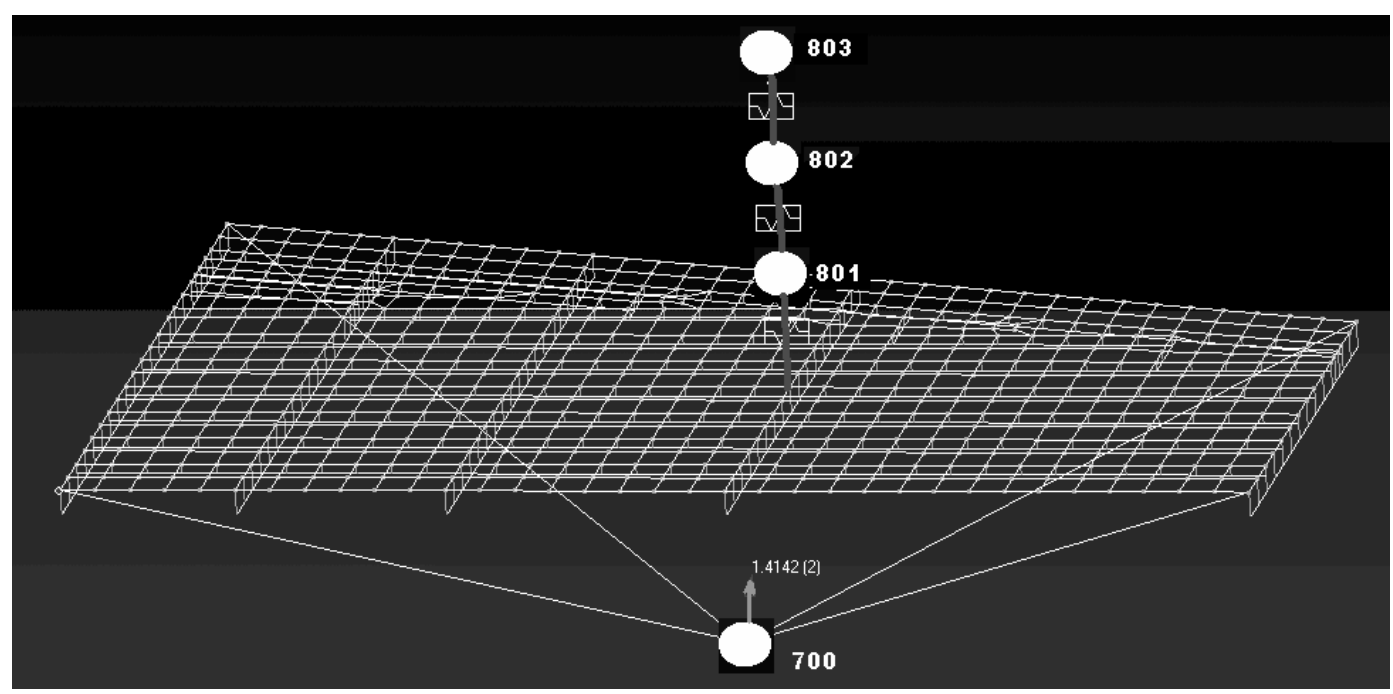

Fig. 10. Model showing the three-mass system. Weights, spring stiffnesses, and dynamic characteristics are listed in Tables 3 and 4.

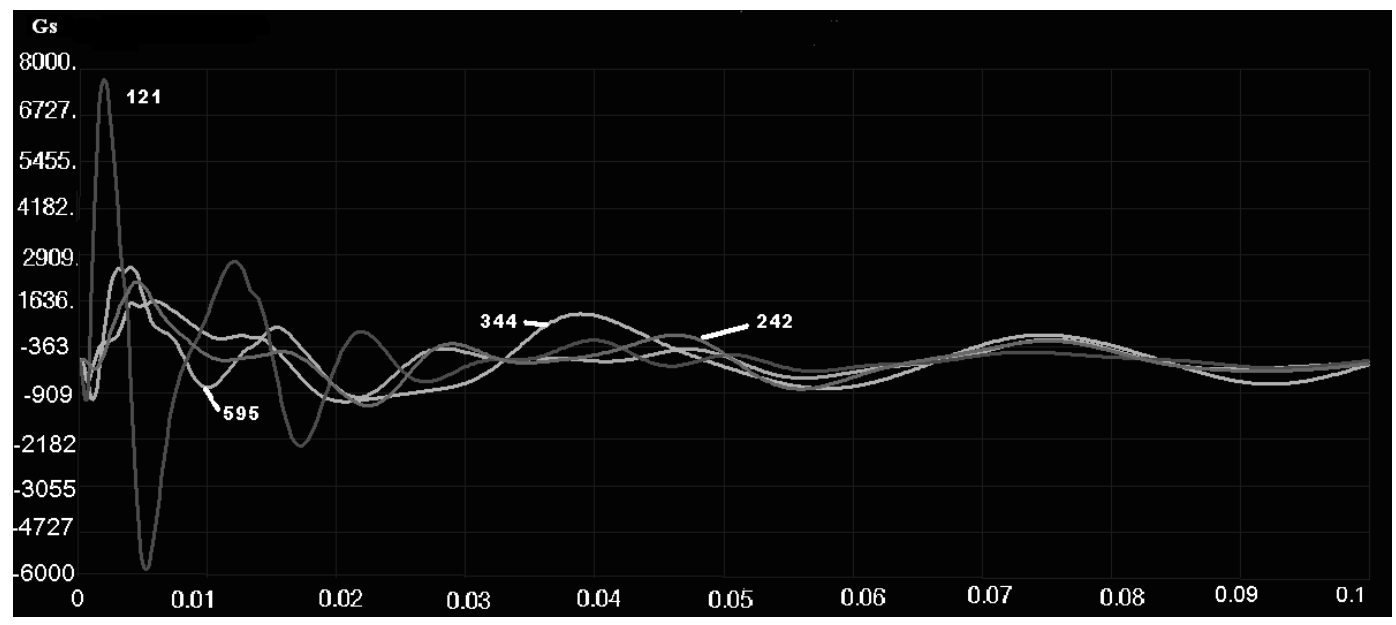

Fig. 11. Calculated accelerations (Gs) of the nodes of the free deck where masses are to be attached (Table 2).

Shock spectra were calculated using UERD Tools [2]. The shock spectrum curves of the free deck motion at Nodes 595, 344, 121 and 242 are plotted in Fig. 13. The effect on the shock spectrum of adding the 1,500 lb mass at Node 121 with a $22 \mathrm{~Hz}$ fixed base frequency is shown in Fig. 14. There is a dip near the fixed-base frequency where the spectrum acceleration is reduced from about $1300 \mathrm{Gs}(303 \mathrm{ft} / \mathrm{sec})$ to about $700 \mathrm{Gs}(163 \mathrm{ft} / \mathrm{sec})$. The change due to adding the 2,000 lb weight at Node 344 with a fixed base frequency of $25 \mathrm{~Hz}$ is shown in Fig. 15. The reduction is larger because the weight is larger and because Node 344 has the largest free deck response. The reduction due to adding the 7,000 lb weight at Node 595 with a fixed-base frequency of $30 \mathrm{~Hz}$ is shown in Fig. 16. The spectrum 


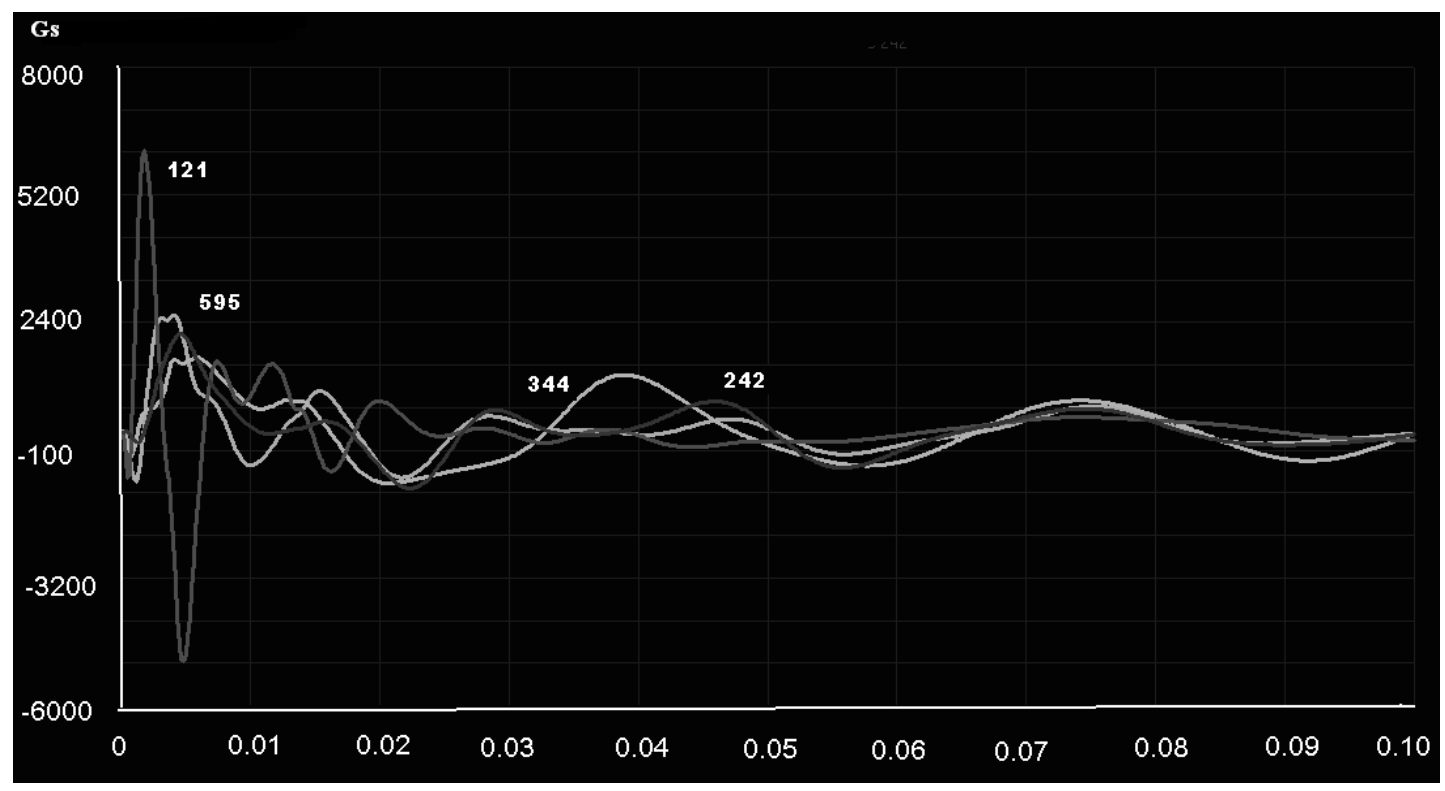

Fig. 12. Calculated accelerations (Gs) of the nodes of the free deck where masses are to be attached except for Node 121 . A $1,500 \mathrm{lb}$ weight is attached at Node 121 with a fixed-base frequency of $22 \mathrm{~Hz}$. Note the reduction in the peak from 7800 Gs to 6000 Gs by comparing results with Fig. 11.

\section{Shock Spectrum}

Free Deck

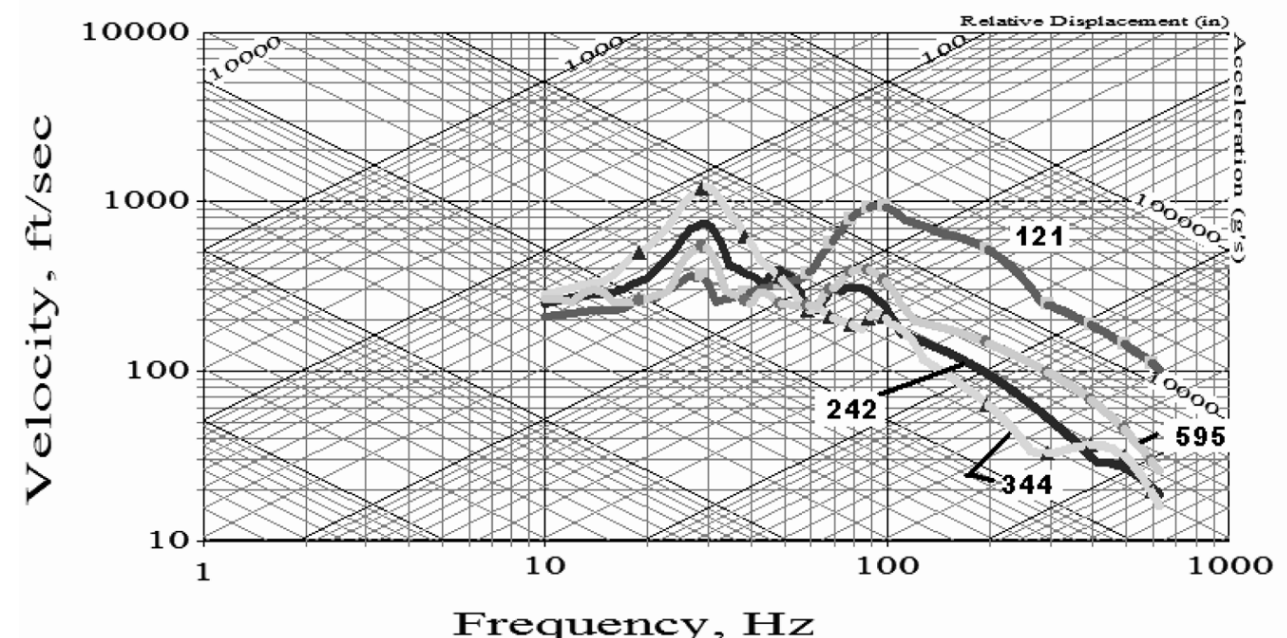

Frequency, $\mathbf{H z}$

\begin{tabular}{|l|}
\hline - Free Deck Motion 242.txtn Text.txt \\
- Free Deck Motion 121.rtfin Text.txt \\
- Free Deck Motion 344 P1ain Text.txt \\
- Free Deck Motion 595.txttn Text.txt \\
\hline
\end{tabular}

Fig. 13. Shock spectrum curves at specified nodes of the free deck. 


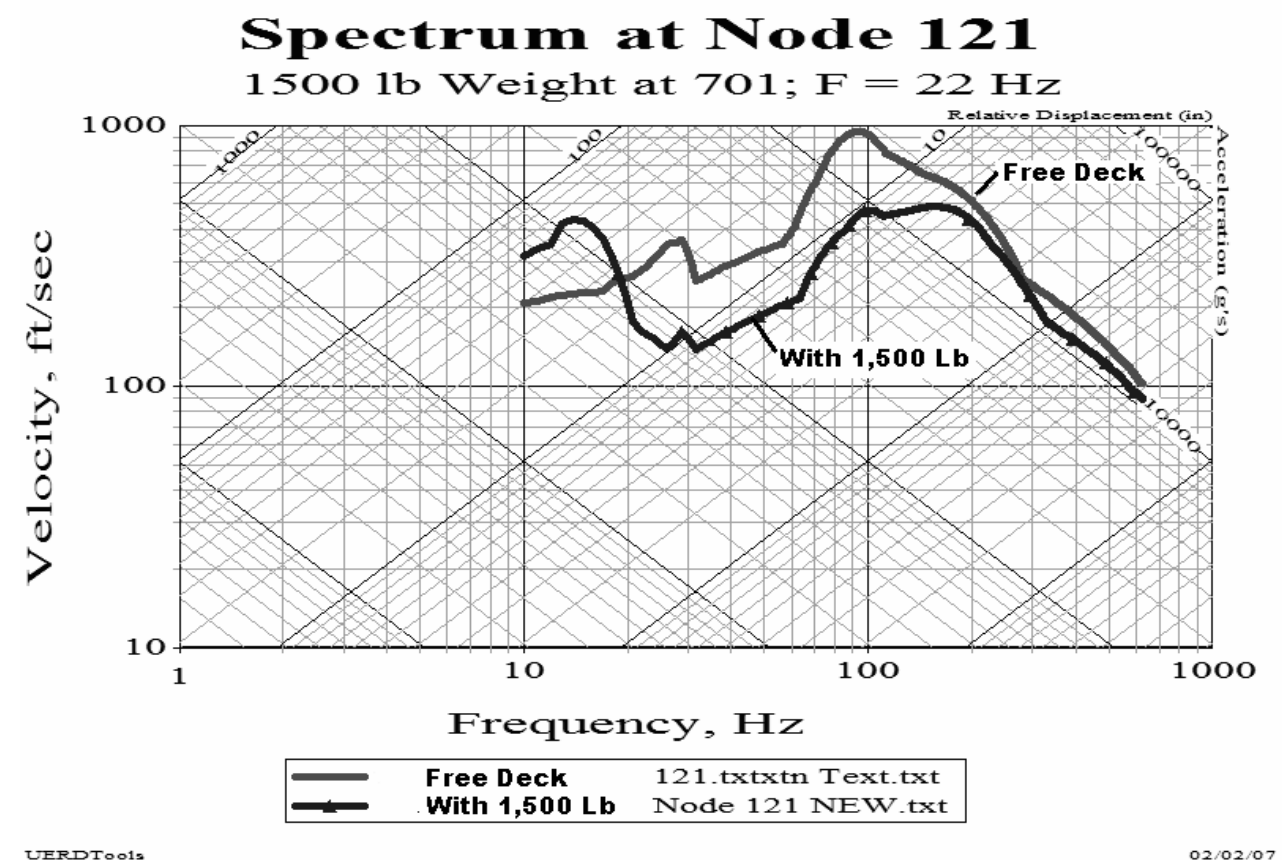

Fig. 14. Shock spectrum curve at Node 121 with a $1,500 \mathrm{lb}$ weight with a fixed-base frequency of $22 \mathrm{~Hz}$ compared to the free deck spectrum curve at the same node. The spectrum dip is obvious.

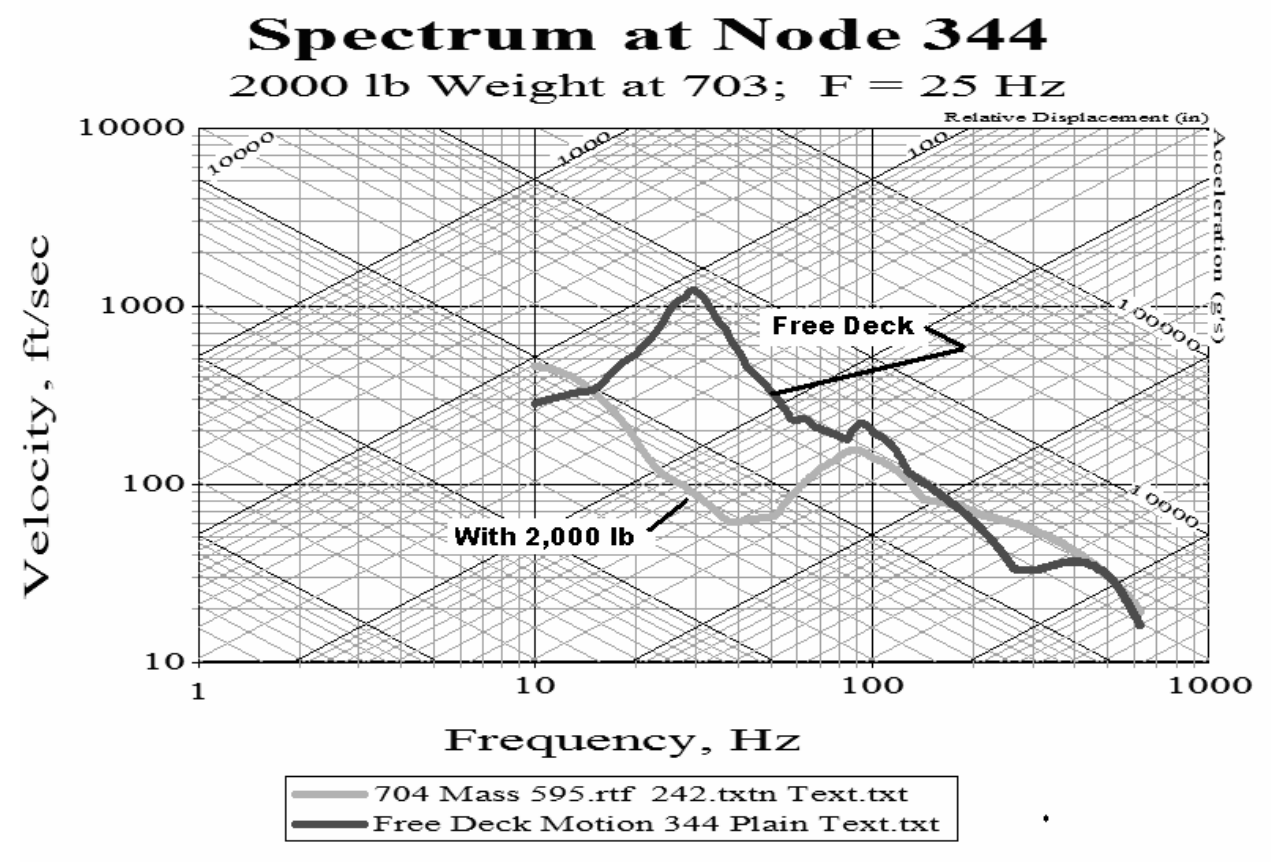

Fig. 15. Shock spectrum curve at Node 344 with a 2,000 lb weight with a fixed-base frequency of $25 \mathrm{~Hz}$ compared to the free deck spectrum curve at the same node. The spectrum dip is obvious. 


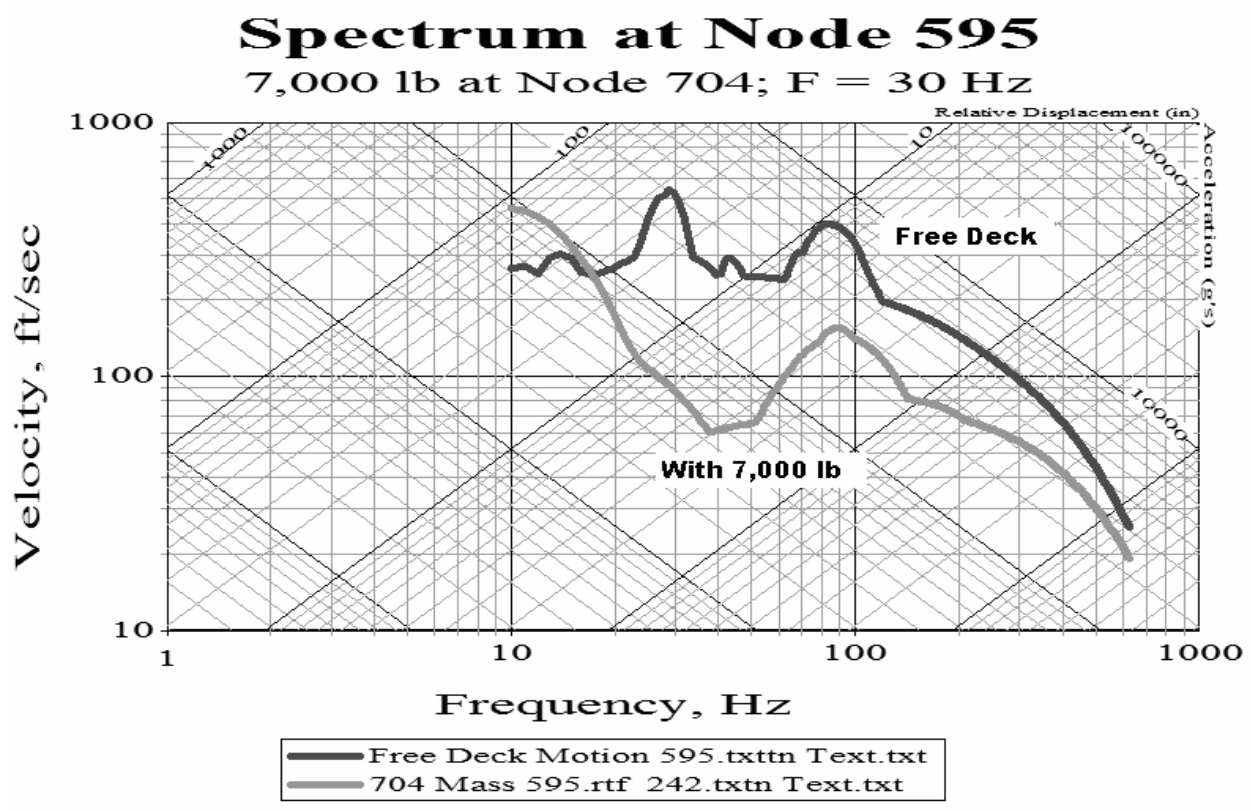

UERDTOO1s

Fig. 16. Shock spectrum curve at Node 595 with a $7,000 \mathrm{lb}$ weight with a fixed-base frequency of $30 \mathrm{~Hz}$ compared to the free deck spectrum curve at the same node. The spectrum dip is obvious.

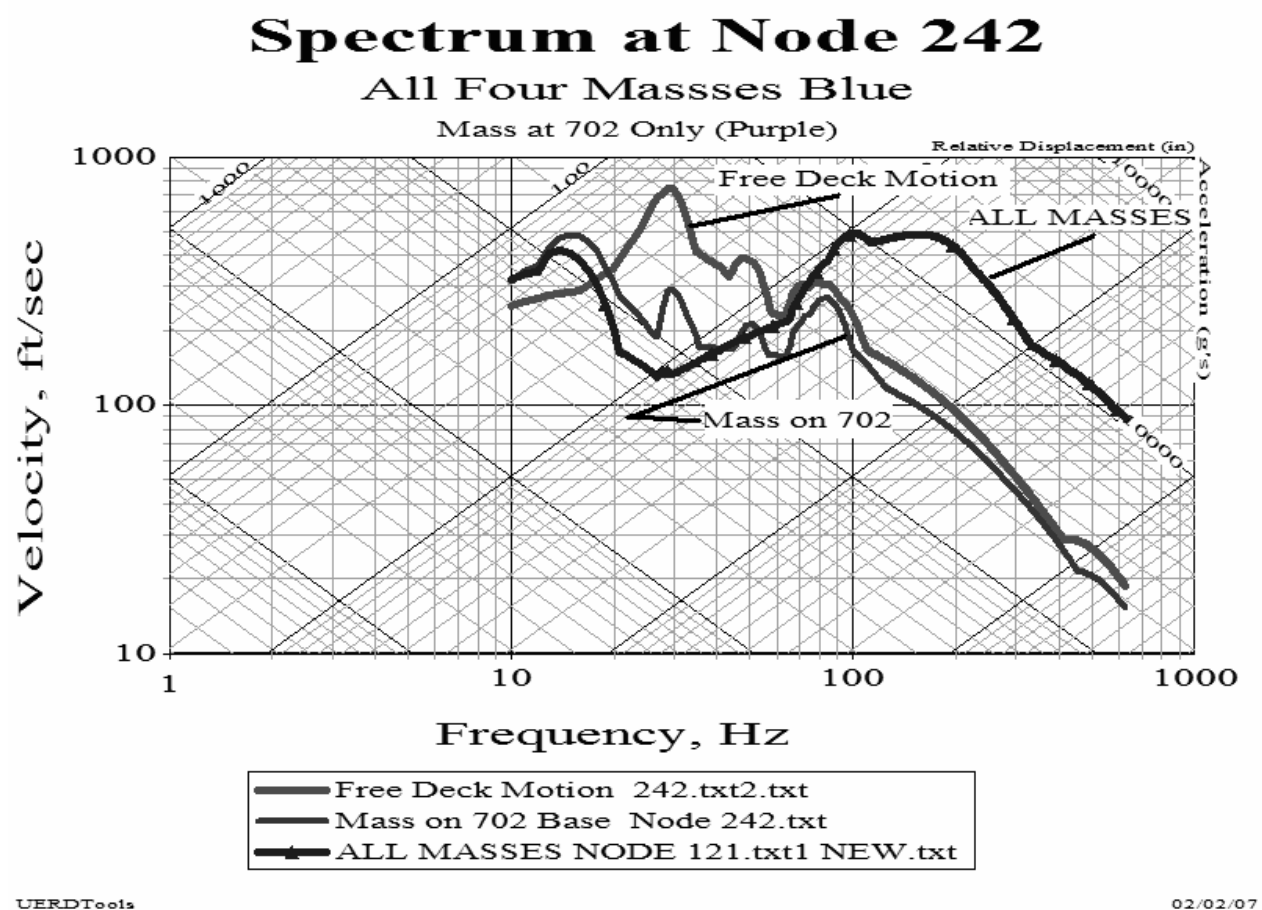

Fig. 17. Shock spectrum curve at Node 242 with a $200 \mathrm{lb}$ weight with a fixed-base frequency of $23 \mathrm{~Hz}$ compared to the free deck spectrum curve at the same node. In addition the spectrum at Node 242 with all dynamic masses attached to the deck is shown in blue. There is a further suppression of the spectrum by the other masses on the deck. 


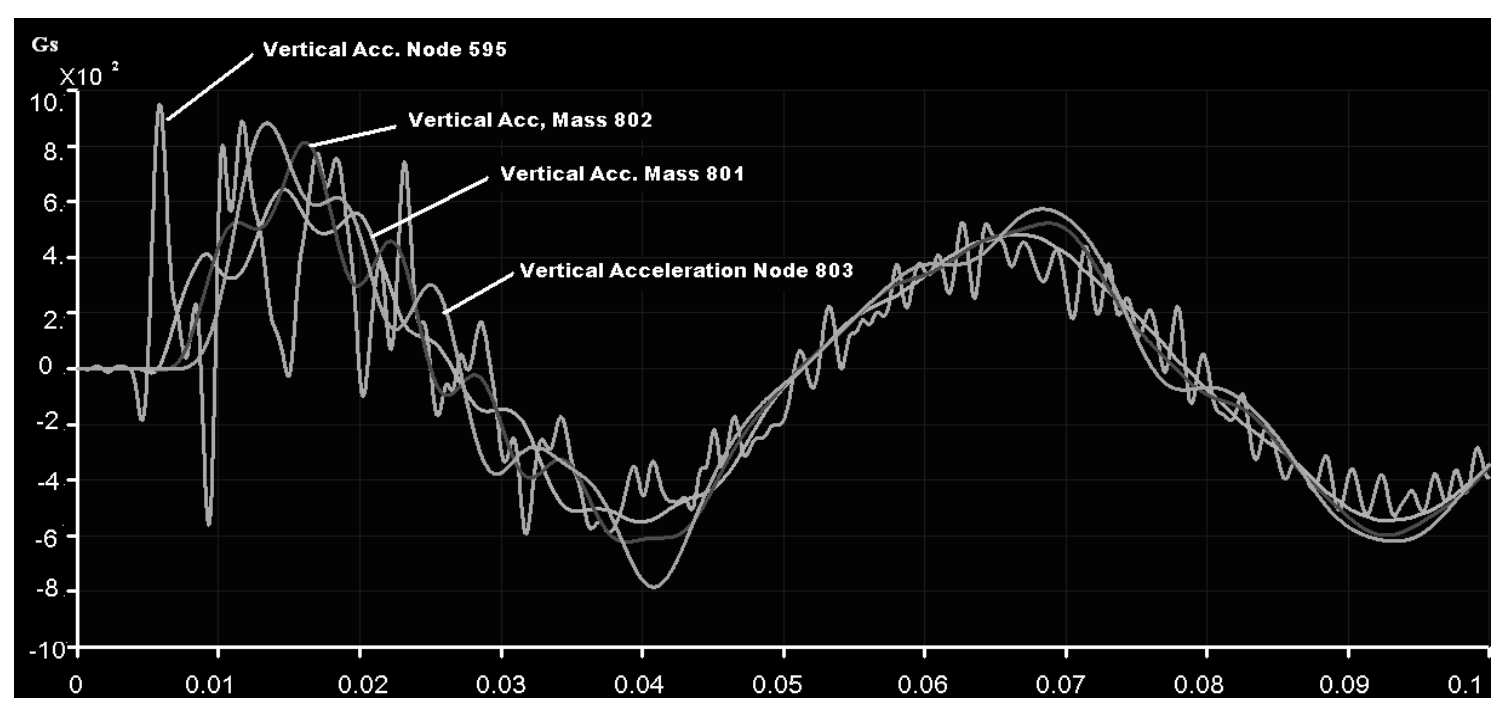

Fig. 18. Time-history accelerations (Gs) of the deck Node 595 for the three-mass system and for the three masses of the system. Nodes 801,802 , and 803 represent the lower, middle, and upper masses of the system, respectively.

comparison from adding the lightest weight at $200 \mathrm{lbs}$ with a fixed base frequency of $23 \mathrm{~Hz}$ at Node 242 is presented in Fig. 17. Even with this light weight acting alone, there is a significant reduction in the spectrum curve.

Also plotted in Fig. 17 is the calculated spectrum curve at Node 242 for the case where all the dynamic masses are attached to the deck. Node 242 is in close proximity to Node 595 with the $7000 \mathrm{lb}$ oscillator. There is a further reduction of the spectrum from $1000 \mathrm{Gs}$ acting alone to $700 \mathrm{Gs}$. The free deck spectrum acceleration at $23 \mathrm{~Hz}$ is near 2250 Gs. Thus the close proximity of a heavy mass to a lighter one reduces the shock spectrum further.

\section{Dynamic calculations - three-mass system}

The three mass system, with a total weight of 7,000 lb shown in Fig. 10 was mounted on the deck at Node 595, which is the same node used for the single DOF 7,000 lb system. The fixed-base frequencies and modal masses are listed in Table 4, above. The frequencies of the three fixed-base system modes are 31, $60 \mathrm{and} 83 \mathrm{~Hz}$, and the modal weights are 5,456 lb, 1,315 lb and $226 \mathrm{lb}$, respectively. Stiffnesses were calculated for single-degree-of-freedom oscillators representing each mode of the three mass system and are listed in Table 4 . The shock spectrum was calculated at Node 595 for the free deck, for the three-mass system, and for the individual oscillators representing Modes 1, 2 and 3.

\subsection{FSP acceleration input to the three-mass system}

Calculated results, to some degree, depend on the size of the time step and the duration of the time-history. The authors repeated their simulations with progressively smaller time-steps until the simulation results converged to an acceptable degree. After some trials the final problems were run with a time step of $0.00002 \mathrm{sec}(0.02$ milliseconds) for a total duration of 0.2 seconds (200 milliseconds). The shock spectra were calculated with UERD Tools [2] using 5\% damping. The time-history velocity curves were trimmed at the largest time in the 200 milliseconds that the velocity went to zero. A step change in the velocity time-history at the end of a velocity curve can lead to errors in the resulting shock spectrum.

The acceleration time-histories are shown in Fig. 18 and the shock spectrum curves are shown in Fig. 19. Shock spectrum curves for the motion at base Node 595 are plotted for the free deck, for the three-mass system and for the systems representing Modes 1-3. These results show that the dynamic interaction of the three-mass system with the deck motion will suppress the spectrum curves over the range of natural frequencies. Furthermore, the reduction for 


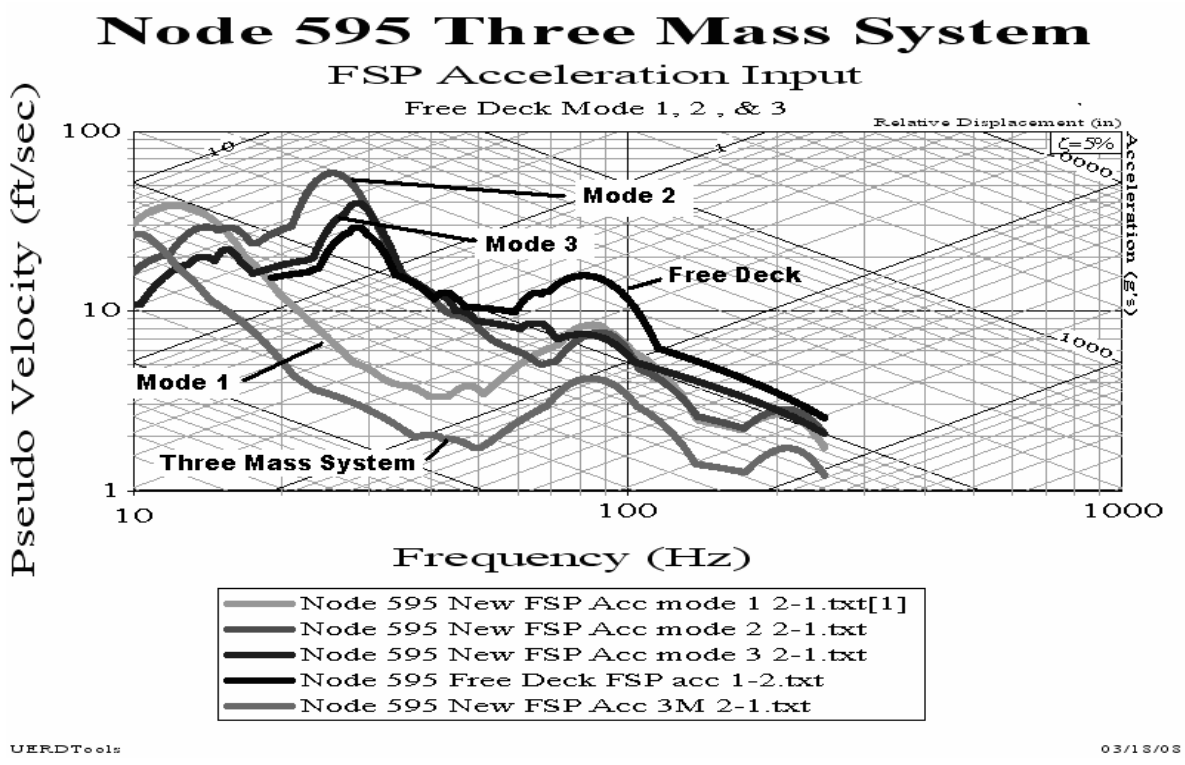

Fig. 19. Shock spectra at Node 595 for the free deck, the three mass system and Modes 1-3 subjected to FSP input. Modal fixed-base frequencies of the system are 31,60 and $83 \mathrm{~Hz}$ respectively.

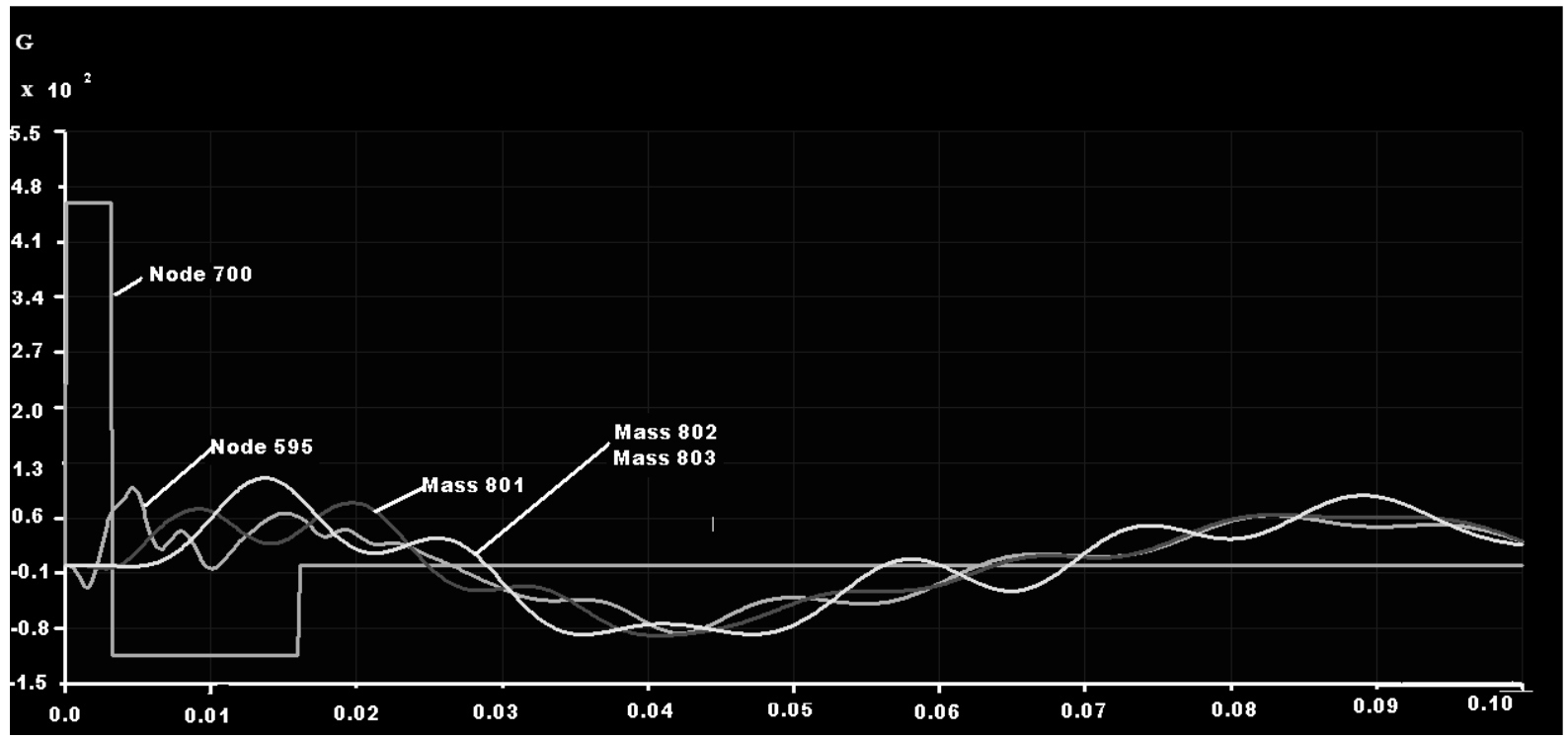

Fig. 20. Time-history accelerations (Gs) of Node 700 and of the masses of the three-mass system subjected to the triangular velocity input. Nodes 801,802 , and 803 represent the lower, middle, and upper masses of the system, respectively. Node 700 , the seismic mass, has a step change in acceleration to develop the triangular velocity plotted in Fig. 2.

the entire system is larger than for the individual modal reductions at their respective fixed-base frequencies. The free deck spectrum pseudo velocity value at $83 \mathrm{~Hz}$ is about $18 \mathrm{ft} / \mathrm{sec}$. With only the third mode single degree-of-freedom system $(83 \mathrm{~Hz})$ on the deck, the pseudo velocity value is reduced to about $7.2 \mathrm{ft} / \mathrm{sec}$. With the entire system present, the spectrum value is further reduced to $4 \mathrm{ft} / \mathrm{sec}$. What these results imply is that the current practice in DDAM of specifying the input to higher modes in a dynamic system may be very conservative when based on the modal weight of that mode alone. Some method must be used to factor in the weight of the entire system to specify the modal spectrum input and not just the modal weight of that mode. 


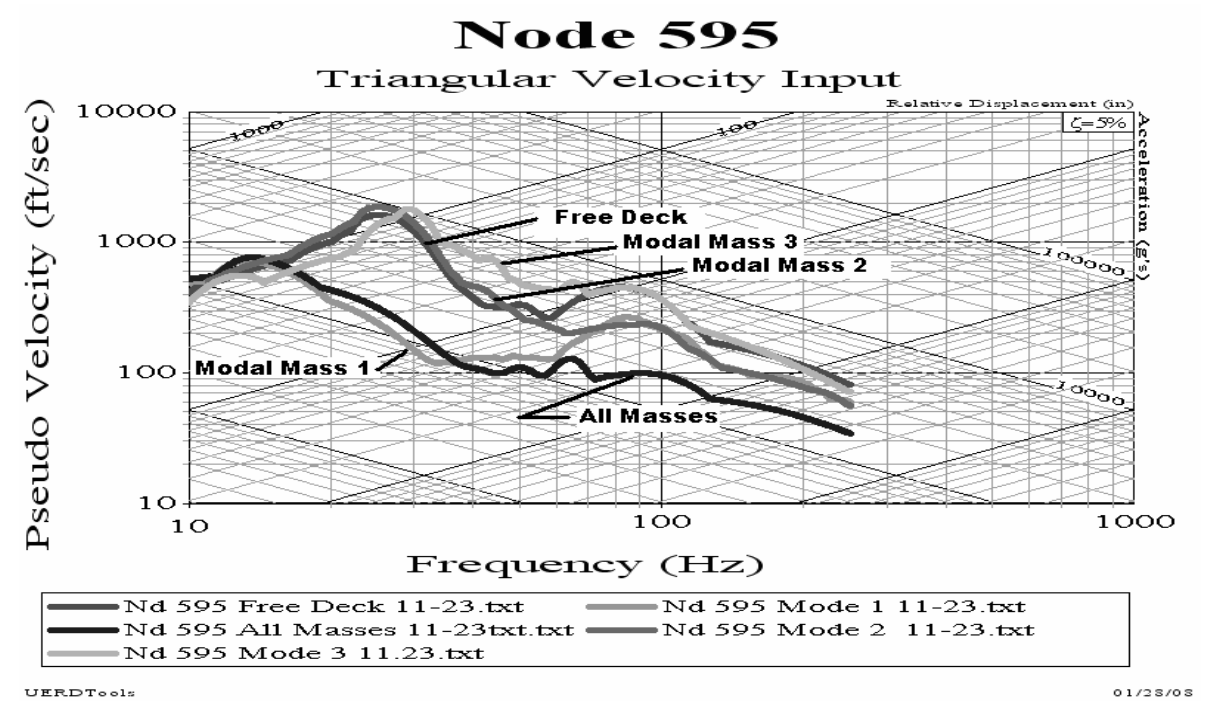

Fig. 21. Shock spectra at Node 595 for the free deck, the three-mass system and Modes 1-3 subjected to the triangular velocity input. Modal fixed-base frequencies of the system are 31,60 and $83 \mathrm{~Hz}$ respectively.

\subsection{Triangular velocity input to the three-mass system}

The acceleration time-history motions of Node 700, Node 595, the mass nodes of the three-mass system (Nodes 801, 802 and 803) are plotted in Fig. 20. The accelerations are in Gs. The resulting shock spectrum curves of the base Node 595 for the free deck, the three-mass system and for each of the three independent modal oscillators are plotted in Fig. 21. As expected, the lowest spectrum response curve occurs when the entire system is present and the highest occurs for the free deck except at the first mode frequency. In this case the deck response with the single-mass system of the first mode at $31 \mathrm{~Hz}$ was slightly lower than the deck motion with the three-mass system at that frequency. However, the spectrum response curves of the second mode $(60 \mathrm{~Hz})$ and the third mode $(83 \mathrm{~Hz})$ are much lower when the entire system is considered then the responses for the single degree-of-freedom systems analyzed one at a time. In fact, the response of the third mode causes no discernable spectrum reduction from the free deck spectrum at the fixed-base frequency of $83 \mathrm{~Hz}$.

\section{Conclusions}

- Dynamic interaction between the structure and equipment base locations must be considered in determining shock spectral inputs.

- The shock spectrum curve may be significantly reduced by dynamic interaction. As the dynamic mass increases the reduction is increased.

- The reduction in the shock spectrum curve is often characterized by a dip in the spectrum at the fixed-base natural frequency or frequencies of the mounted structures. However, the spectrum reduction can occur over a relatively wide frequency range.

- Even a relatively light dynamic system can cause a shock spectrum reduction and/or dip at the fixed-base frequency.

- The shock spectrum of a light system can be further reduced by a heavy system mounted on a deck in close proximity to the light system. This reduction has implications to a multi-mass system with many higher modes involving small modal masses.

- A three-mass system was subjected to two different shock inputs: a triangular velocity pulse and an FSP shock motion. In addition each mode was modeled as a single-degree-of-freedom system with its modal mass and the spring stiffness that results in the modal fixed-base frequency. 
- The most significant result observed for both shock inputs is that the base shock motion as determined by the shock spectrum at the frequencies of the higher two modes is much less in the spectrum curves of the entire system as compared to the shock spectra of the individual modal responses. This result implies that the DDAM procedure of determining the shock input of the higher modes in a large dynamic model may subject the system to much too high of a shock input and is excessively conservative.

- Shock design problems may be predicted where none exist.

- At this time the authors did not attempt to develop a recommendation of a rational method of determining an effective modal mass of higher modes in a large system. More analytical work, evaluation of existing experimental data and, perhaps, experimental work is needed.

- Dynamic interaction or the "spectrum-dip" will vary depending on the relative frequencies between the dynamic system and input motion. For stiff bases vibrating at low frequencies, the effect is reduced for a given excited system. Not all combinations are examined but some of this effect can be seen in the spectrum curves presented. The largest interaction normally occur at the fixed-base frequencies of the structure.

\section{Acknowledgement}

The authors are grateful to the technical staff of Noran Engineering for their support in this study and to Engineering Consultants Group, Inc. of Akron, Ohio, for the use of NEiNastran Modeler and NEiNastran Editor. Shock spectrum curves were calculated using UERD Tools developed by Paul Mantz et al. [2].

\section{References}

[1] P.F. Cunniff and R.P. Collins, Structural interaction effects on shock spectra, The Journal of the Acoustical Society of America 43(2) (February 1968), 239-244.

[2] P. Mantz, F. Costanzo, J. Howell III, D. Ingler, E. Luff and S. Okano, UERD Tools, 4.4, NSWCCD, 29 August, 2007.

[3] N.M. Newmark and E. Rosenblueth, Fundamentals of Earthquake Engineering, Prentice-Hall, 1971.

[4] G.J. O'Hara, Effect on shock spectra of dynamic interaction of structures, NRL Report 5236, 16 December, 1958.

[5] G.J. O'Hara, Shock spectra and shock design spectra, Naval Research Laboratory, NRL Report 5386, November 1959.

[6] R.J. Scavuzzo and D.D. Raftopoulos, An analysis of spectrum dip in underwater shock, The Shock and Vibration Bulletin 40 (1970).

[7] R.J. Scavuzzo, J.L. Bailey and D.D. Raftopoulos, Lateral structure interaction with seismic waves, Journal of Applied Mechanics (March 1971).

[8] R.J. Scavuzzo and H.C. Pusey, Naval shock analysis and design, Shock and Vibration Monograph 17 (SVM-17), the Shock and Vibration Information Analysis Center, HI-TEST Laboratories, Inc., Arvonia, VA, 2007

[9] I. Vigness, Instrumentation, analysis, and problems concerning shock and vibration, Structural Mechanics - Proceedings of the First Symposium on Naval Structural Mechanics, Pergamon Press, Inc., 1960, pp. 506-532. 

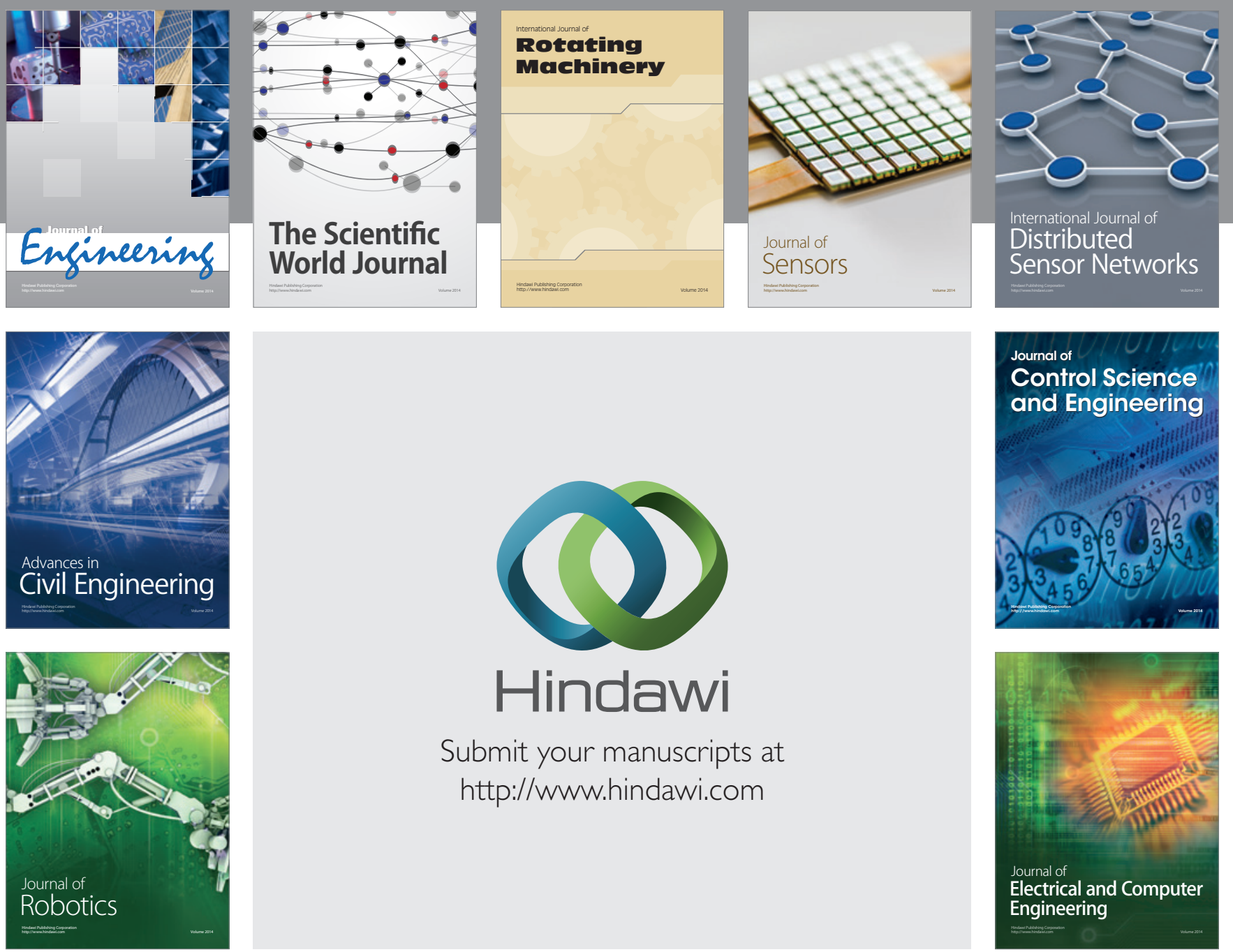

Submit your manuscripts at

http://www.hindawi.com
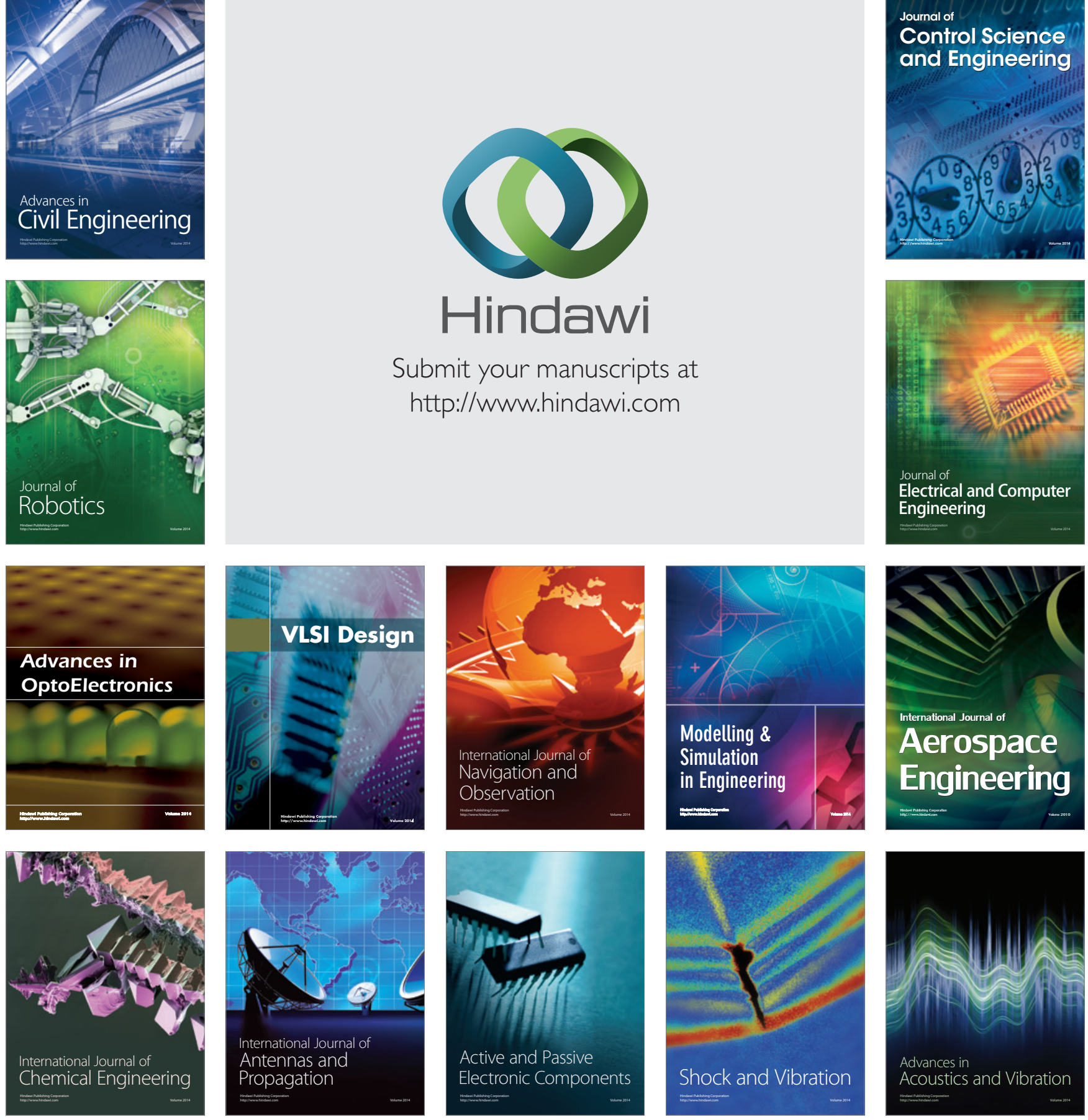